\title{
nature/methods
}

\section{Supplement on single-cell analysis}

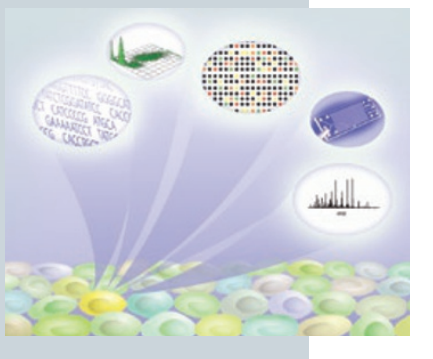

The cover image depicts artistically the isolation and analysis of a single cell from within a heterogenous cellular population. Cover design by Erin Dewalt. iological systems are complex, and the cells that comprise them are, more often than not, molecularly and functionally distinct. This is true for the cells that make up a tissue in a multicellular organism - the highly heterogenous neurons of the human brain, for instance-but also for isogenic single-celled bacteria and for mammalian cells in vitro. Stem cells, in particular, are increasingly recognized as being divisible into subpopulations that, at least in some cases, have distinct functional properties.

Cellular heterogeneity may come about for several nonexclusive reasons: because of genetic or epigenetic differences, as a consequence of differing microenvironments or because there is a stochastic component to the molecular processes occurring in otherwise identical cells. Whatever the source, there are many circumstances in which heterogeneity is an inherent and also a desirable property of cells.

Many experimental analyses, however, are carried out on pools of cells and are consequently blind to heterogeneity in the population. Measurements of averages, by definition, smooth the differences out! One route to a finer-grained picture of complex biological systems is to make measurements on single cells. But whereas methods such as electrophysiology or high-resolution imaging intrinsically lend themselves to analysis of single cells, others-biochemical approaches to examine gene expression, protein levels or smallmolecule distributions, for instance-must perform at very high sensitivity to measure the small quantities of material that can be extracted from a single cell.

Such measurements are beginning to be possible. An online supplement brings together three Reviews and a Commentary discussing methods for reading out genomes and for measuring transcript, peptide and metabolite levels in a single cell. Two Perspectives also describe methods for the study of single stem cells. In the following pages, we summarize the contents of these papers. To read the supplement itself, please visit our website.

We hope this collection is both practically useful and also stimulates thinking about the nature of biological heterogeneity and the ways in which single-cell analysis can help decipher it.

\section{Natalie de Souza}

\section{ONLINE CONTENTS}

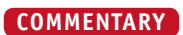

S2 Single-cell genomics Tomer Kalisky \& Stephen R Quake

\section{REVIEWS}

S6 Development and applications of single-cell transcriptome analysis

Fuchou Tang, Kaiqin Lao

\& M Azim Surani

S12 Validating transcripts with probes and imaging technology Shalev Itzkovitz \& Alexander van Oudenaarden

\section{S20 Profiling metabolites} and peptides in single cells Stanislav S Rubakhin,

Elena V Romanova, Peter Nemes

\& Jonathan V Sweedler

\section{PERSPECTIVES}

S30 Long-term singlecell imaging of mammalian stem cells

Timm Schroeder

S36 Clonal interrogation of stem cells

Kristin Hope

\& Mickie Bhatia
Editor, Nature Methods Daniel Evanko Managing Production Editor

Supplement Editor Natalie de Souza

Publisher Stephanie Diment

Senior Copy Editor Irene Kaganman
Ingrid McNamara

Production Editor Amanda Crawford

Marketing Joanna Budukiewicz 